\title{
Systematic review and pathway enrichment analysis of Chinese medicine in preventing recurrence and improving prognosis of cholelithiasis after gallbladder-preserving lithotripsy
}

\author{
Bo Li ${ }^{1 \#}$, Yiyi Lin ${ }^{2 \#}$, Bo Nie ${ }^{2}$, Guozhen Zhao ${ }^{1}$, Xiatian Zhang ${ }^{3}$, Mengting Zhu ${ }^{2}$, Jing Hu ${ }^{1}$, Po Huang ${ }^{1}$, \\ Shuo Feng ${ }^{1}$, Ruijin Qiu ${ }^{2}$, Guihua Tian ${ }^{2}$, Shengsheng Zhang ${ }^{1}$, Hongcai Shang ${ }^{2}$ \\ ${ }^{1}$ Beijing Hospital of Traditional Chinese Medicine, Capital Medical University, Beijing Institute of Traditional Chinese Medicine, Beijing, China; \\ ${ }^{2}$ Beijing Evidence-based Chinese Medicine Center, Dongzhimen Hospital, Beijing University of Chinese Medicine, Beijing, China; ${ }^{3}$ School of \\ Mathematical Sciences, University of Southampton, Southampton, UK \\ Contributions: (I) Conception and design: Y Lin, X Zhang, B Nie, G Tian, B Li; (II) Administrative support: B Nie, G Tian, B Li, H Shang; (III) Provision \\ of study materials or patients: X Zhang, M Zhu, G Zhao, J Hu, P Huang, S Feng, R Qiu; (IV) Collection and assembly of data: Y Lin, M Zhu, B Li; (V) \\ Data analysis and interpretation: Y Lin, X Zhang, G Zhao, B Li; (VI) Manuscript writing: All authors; (VII) Final approval of manuscript: All authors. \\ "These authors contributed equally to this work. \\ Correspondence to: Guihua Tian. Dongzhimen Hospital, Beijing University of Chinese Medicine, Beijing 100700, China. Email: rosetgh@163.com; Bo \\ Li. Beijing Hospital of Traditional Chinese Medicine, Capital Medical University, Beijing Institute of Traditional Chinese Medicine, Beijing 100010, \\ China. Email: libo@bjzhongyi.com; Shengsheng Zhang. Beijing Hospital of Traditional Chinese Medicine, Capital Medical University, Beijing \\ Institute of Traditional Chinese Medicine, Beijing 100010, China. Email: zhss2000@163.com; Hongcai Shang. Dongzhimen Hospital, Beijing \\ University of Chinese Medicine, Beijing 100700, China. Email: shanghongcai@foxmail.com.
}

Background: Traditional Chinese medicine (TCM) may improve the prognosis management of cholelithiasis patients after gallbladder-preserving lithotripsy. To explore the evidence for this view, we systematically reviewed the efficacy and safety of TCM for improving the prognosis of cholelithiasis after gallbladder-preserving lithotripsy and performed functional pathway enrichment analysis of TCM target genes.

Methods: In this systematic review (SRs), we searched six Chinese or international databases to collect randomized controlled clinical trials (RCTs) of TCM in preventing the recurrence of cholelithiasis after gallbladder-preserving lithotripsy. The literature was independently screened by 2 reviewers, who then extracted the data. The Cochrane risk-of-bias and Grading of Recommendations, Assessment, Development and Evaluations (GRADE) tools were used to assess the included studies' risk of bias and quality of evidence, respectively. And, the Gene Ontology (GO) and Kyoto Encyclopedia of Genes and Genomes (KEGG) pathway enrichment analyses would be conducted on the TCM prescriptions in the included literature to find the effective component and mechanism of TCM in the prognosis management of gallbladderpreserving lithotripsy. Analysis in this research would be conducted by R 3.5.2 software.

Results: A total of 1,024 articles were retrieved, and 9 RCTs involving 926 participants were included after the step-by-step screening. The risk of bias for each important outcome in all the studies was "uncertain". The meta-analysis showed that compared with blank control, TCM prevented cholelithiasis by decreasing the recurrence rate, complications incidence, gallbladder wall thickness, and gallbladder contraction degree. But, there were no significant differences in the rate of the adverse reaction. The result of the GO and KEGG analysis revealed that the mechanism of prevention of TCM in gallstone recurrence may be related to the cholesterol metabolic pathway and that naringin from Glycyrrhiza may be the effective component in the prevention of recurrence.

Conclusions: Existing evidence suggests that the use of TCM may reduce the recurrence rate after gallbladder-preserving lithotripsy and this effect may be related to the flavonoid glycoside naringin from Glycyrrhiza uralensis, but more RCTs with high quality in this area may be needed to have a robust conclusion. 


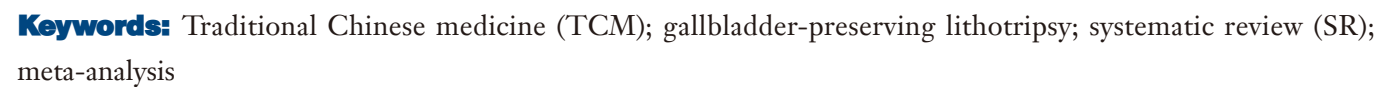

Submitted May 19, 2020. Accepted for publication Jun 18, 2021.

doi: 10.21037/apm-20-1135

View this article at: https://dx.doi.org/10.21037/apm-20-1135

\section{Introduction}

With the increasing prevalence of risk factors for cholelithiasis in the Chinese population, such as habitual meat-eating, overweight, and obesity (1-3), the incidence of cholelithiasis in China is increasing annually and doubling every 10 years (4). In 2012, the incidence of cholelithiasis among urban residents in China was approximately $4.6 \%$ (5). Currently, the main treatment methods for cholelithiasis include cholecystectomy, oral litholysis, and extracorporeal shockwave lithotripsy (6). Cholecystectomy has been the main standard for the treatment of cholecystolithiasis for a long time (7); however, with the popularization of choledochoscope in hospitals, minimally-invasive gallbladder-preserving lithotripsy has been gradually adopted throughout China (8). Compared with cholecystectomy, it reduces side effects like the loss of bile storage and increases the detection of choledocholithiasis (9). At the same time, effective postoperative medical care can also assist with patient recovery (10). At present, traditional Chinese medicine (TCM) is commonly used to treat patients after gallbladder-preserving lithotripsy. Clinical observation and evidence have suggested that the recurrence rate of gallstones after minimally invasive gallbladder-preserving lithotripsy may be reduced when TCM treatment is used postoperatively (11). However, the sensitivity of literature retrieval was poor, and there has been a lack of discussion on complications, safety, and other outcome indicators. In this study, a meta-analysis was performed to review the efficacy and safety of TCM for clinically preventing recurrence after gallbladder-preserving lithotripsy and to provide evidence for further development of TCM for this purpose.

We present the following article in accordance with the PRISMA reporting checklist (available at https://dx.doi. org/10.21037/apm-20-1135).

\section{Methods}

\section{Inclusion and exclusion criteria}

The inclusion criteria were as follows: (I) RCTs comparing Chinese herbs for preventing the recurrence of cholelithiasis after gallbladder-preserving surgery; (II) laparoscopic minimally invasive or open gallbladder-preserving surgery was used to treat patients with cholecystolithiasis who met the diagnostic criteria of the Chinese Consensus on Diagnosis and Treatment of Chronic Cholecystitis and Gallstones (Shanghai, 2014) (12). The inclusion of patients was not limited by race, nationality, or disease course; (III) the experimental group was treated with TCM, with no restrictions on type or efficacy. The control group was given blank control intervention; (IV) the main outcome indicators were: (i) recurrence rate and (ii) physiological indicators: gallbladder wall thickness, and gallbladder contractility degree. The secondary outcome indicator was (i) safety.

The exclusion criteria were as follows: (I) study included patients with cholecystolithiasis treated by cholecystectomy; and (II) study included patients who had been treated with Western medicine after gallbladder-preserving lithotripsy.

\section{Retrieval strategy}

Electronic searches of the Chinese Biomedical Literature Database (CBM), Chinese Scientific Journal Database (VIP), WanFang, Chinese National Knowledge Infrastructure (CNKI), Ovid MEDLINE (R), Embase (1946 to January Week 3 2019), and EBM Reviews-Cochrane Central Register of Controlled Trials (CCTR) (December 2018) databases were performed. RCTs of TCM intervention after gallbladder-preserving lithotripsy were collected. The retrieval time was from database establishment to 17 November 2018. References of the retrieved studies were searched to supplement the included literature. The search was conducted using a combination of subject words and free words. English search terms and Chinese search terms included: "gallstones", "TCM", "drugs", "Chinese herbal medicine", and "oriental medicine". The specific retrieval strategy is shown in Table 1, using Ovid MEDLINE (R) as an example.

\section{Literature screening and data extraction}

The literature was independently screened by 2 reviewers 
Table 1 Ovid MEDLINE(R) retrieval strategy

\#1 exp Gallstones
\#2 exp Drugs, Chinese Herbal
$\# 3$ exp TCM
$\# 4$ \#2 OR \#3
$\# 5$ \#1 AND \#4

(LY and $\mathrm{ZM}$ ) who extracted and cross-checked the data. Any disagreements between the reviewers were resolved through consultation with a $3^{\text {rd }}$ reviewer (LB), and any missing information was retrieved by contacting the author where possible. When selecting documents, the titles and abstracts were read first. After the exclusion of obviously unrelated documents, the full texts were read to further determine studies' suitability for inclusion. The data extracted mainly included: (I) basic study information, including research topics, first authors, magazines, and time of publication; (II) baseline characteristics of the participants, including sample number of each group, age, sex, and disease status; (III) specific details of interventions and follow-up time; (IV) critical elements of bias risk assessment; and (V) outcome indicators and measurement data concerned: recurrence rate, incidence of complications, gallbladder wall thickness, gallbladder contraction degree, and safety. The included studies were eligible for synthesis.

\section{Bias risk assessment}

The 2 reviewers, $L Y$ and $Z M$, evaluated the bias risk of the RCTs according to the Cochrane bias risk assessment tool (13). If disagreement arose, LB was consulted to reach a consensus. The risk of bias was assessed for each important outcome of each study, and the studies were classified as follows (14): (I) low bias risk: all items were assessed as having a low risk of bias; (II) uncertain: 1 or more items were assessed as having an uncertain risk of bias; (III) high bias risk: 1 or more items were assessed as having a high risk of bias.

\section{Evidence GRADE evaluation}

Based on the results of the systematic evaluation, the Grading of Recommendations, Assessment, Development and Evaluations (GRADE) system was used for the evaluation of evidence grade (15). The quality of evidence was classified as follows (16,17): (I) high quality (we were extremely sure that the real effect value was close to the estimated value of the effect); (II) medium quality (we had moderate confidence in the estimated value of the effect; the true value may have been close to the estimated value, but different possibilities remained between them); (III) low quality; (we had limited confidence in the estimated value of the effect; the true value may have been extremely different to the estimated value); and (IV) very low quality (we had little confidence in the estimated value of the effect; the true value may have been extremely different from the estimated value).

\section{Analysis of active components of specific drugs and target pathway annotation}

According to the TCM systems pharmacology database and analysis platform (TCM-SP), the active ingredients [oral bioavailability (OB)\% $>30 \%$, drug-likeness (DL) $>0.18]$ and the corresponding validated targets of TCM$\mathrm{SP}$ were included. Conforming to the DisGeNET discovery platform, the targets associated with gallstones were selected. The search terms included: "cholehiasis", "cholecystolithiasis", "cholesterol gallstones", and "pigment gallstones".

Based on the relationships among components of TCM, single-drug components, disease targets, and targets, an active component-target network was established. A protein-protein interaction (PPI) network was built using TCM treatment targets. The Cytoscape 3.2.1 (https:// cytoscape.org) software was used for network visualization, and key components were selected according to the number of nodes. Gene Ontology (GO) and Kyoto Encyclopedia of Genes and Genomes (KEGG) pathway enrichment analyses of effective components and disease co-regulatory genes were carried out with R 3.5.2 software (https://www.cran. r-project.org/).

\section{Statistical analysis}

The meta-analysis was performed with $\mathrm{R} 3.5 .2$ software and was visualized by forest plots. Relative risk (RR) was used as the effect indices for recurrence rate and incidence of complications. Mean difference (MD) was used as the effect indices for gallbladder wall thickness and degree of gallbladder contraction. Risk difference (RD) was used as the effect indices for adverse reactions. Point estimates and $95 \%$ confidence intervals (CIs) were calculated for 


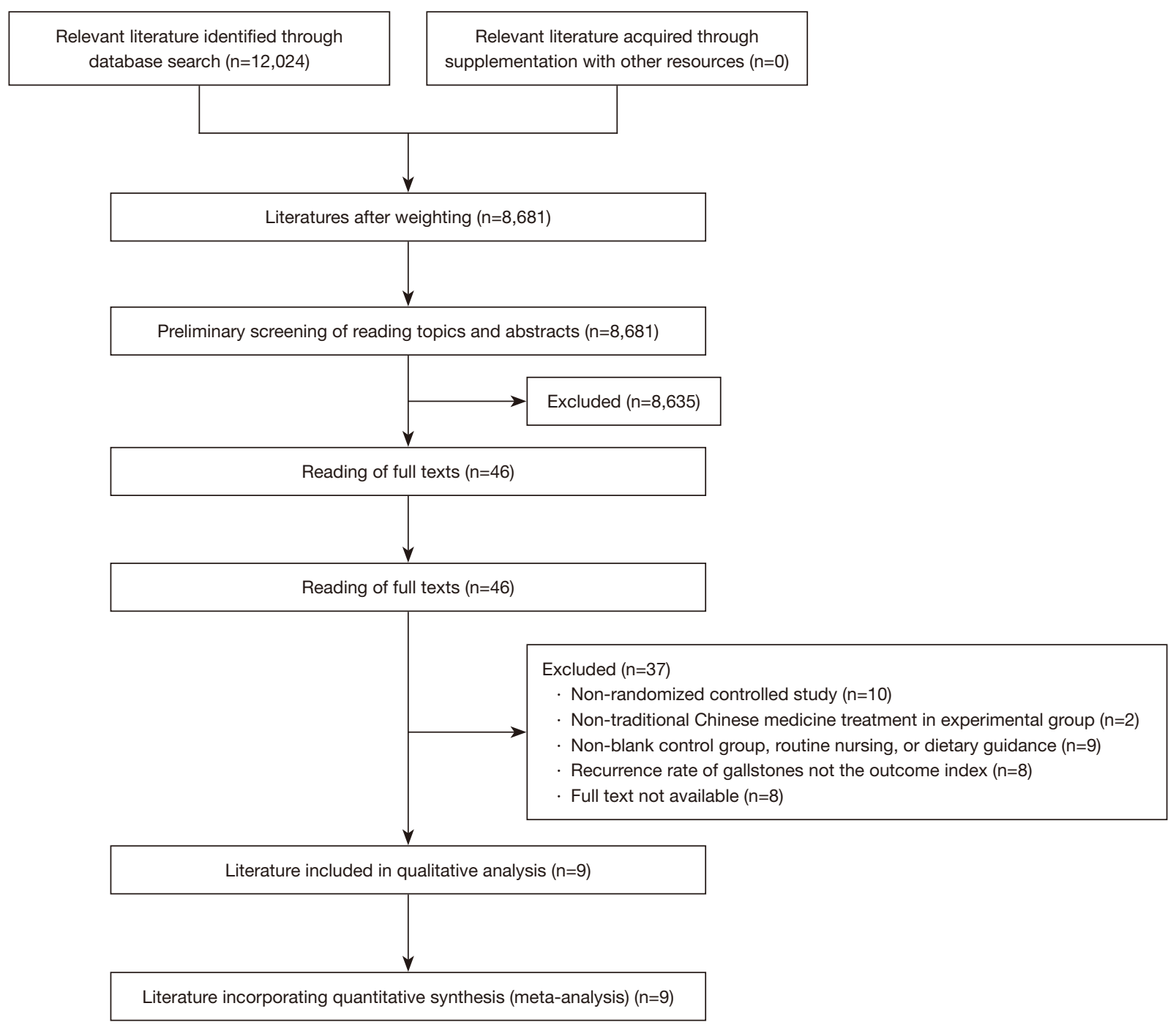

Figure 1 Literature screening process and results. The number of documents detected on the databases searched was as follows: CBM $(\mathrm{n}=3,084)$, CNKI ( $\mathrm{n}=1,044)$, VIP ( $\mathrm{n}=2,736)$, WanFang Data $(\mathrm{n}=5,095)$, Ovid MEDLINE(R) ( $\mathrm{n}=30)$, Embase ( $\mathrm{n}=34)$, and CCTR ( $\mathrm{n}=1)$.

each effect. Heterogeneity among the results was analyzed by chi-square $\left(\chi^{2}\right)$ test (the test level was alpha $=0.1$ ), and the heterogeneity was quantitatively judged by $\mathrm{I}^{2}$. If no statistical heterogeneity existed among the results, the fixed-effects model was used to carry out the meta-analysis. If statistical heterogeneity existed among the results, further analysis to identify the source of heterogeneity was conducted; after the sources of obvious clinical heterogeneity had been excluded, the random-effects model was used to perform the meta-analysis. Obvious clinical heterogeneity was investigated through subgroup or sensitivity analysis, or descriptive analysis alone. Funnel plots were used to evaluate the risk of bias across studies.

\section{Results}

\section{Literature screening process and results}

A total of 12,024 related studies were initially retrieved, and 9 RCTs (18-26) involving a total of 926 patients were eventually included after step-by-step screening. The literature screening process is shown in Figure 1.

\section{Basic characteristics of the included studies and bias-risk assessment results}

Basic characteristics of the included studies are shown in Table 2, and the results of the bias risk assessment are shown 
Table 2 Basic characteristics of the included studies

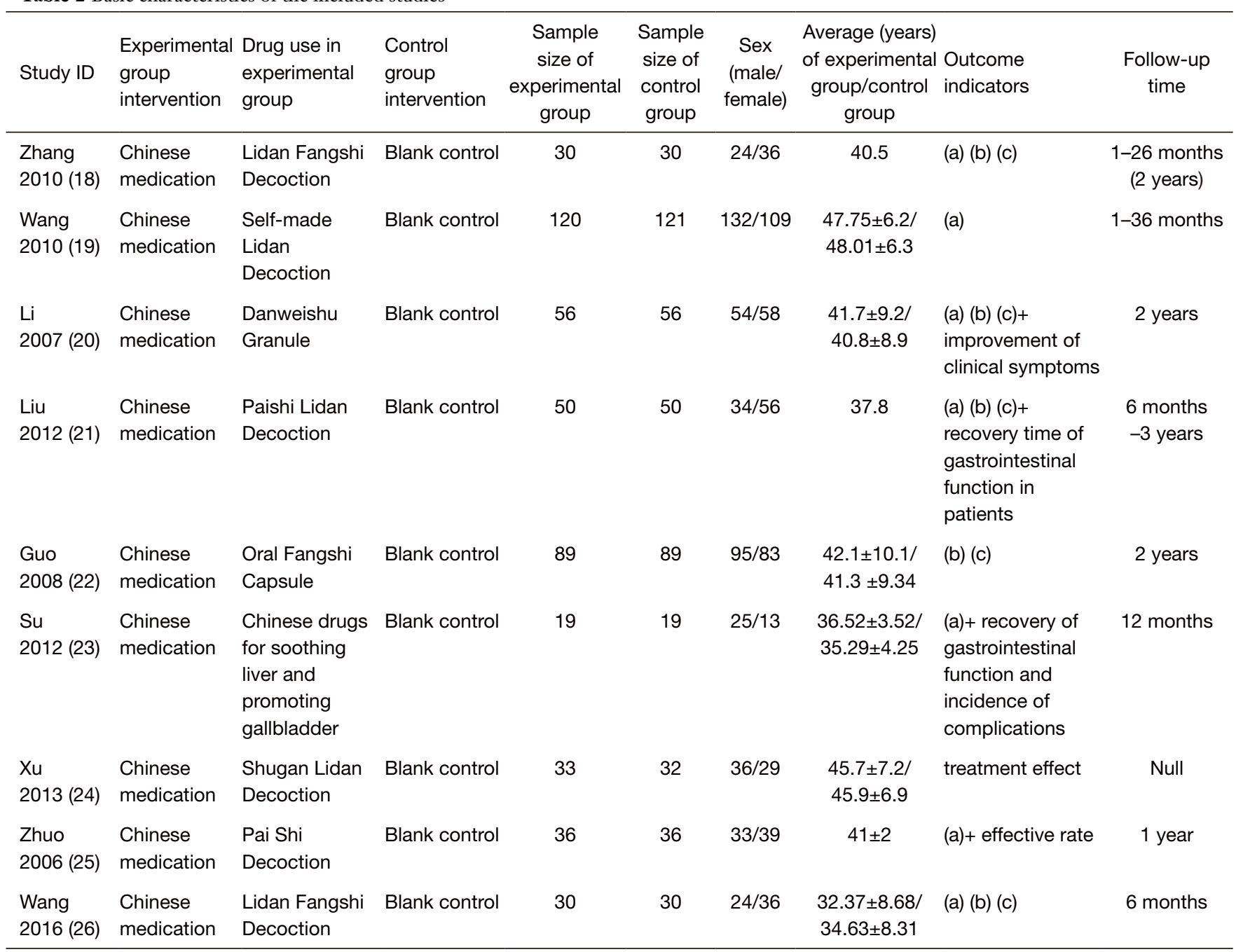

(a) Recurrence of gallstones; (b) gallbladder wall thickness; (c) gallbladder contraction function.

in Table 3. The bias risk of each important outcome in all studies in the meta-analysis was "uncertain". A summary of the bias risk of the included studies is shown in Figure 2. The preventive effect of specific interventions on recurrence events is shown in Figure 3.

\section{Meta-analysis results}

\section{Recurrence rate}

Nine RCTs, involving 901 cases, compared the recurrence rate between patients who received TCM and patients who received a blank control. Meta-analysis using the fixedeffects model showed that there was a significant difference between the TCM group and the blank control group
$(\mathrm{RR}=0.32,95 \%$ CI: 0.22 to $0.56, \mathrm{P}<0.01)$ (Figure 4$)$.

\section{Incidence of complications}

One RCT, involving 38 cases, compared the incidence of complications between patients who received TCM and patients who received a blank control. Meta-analysis using the fixed effects model revealed a significant difference between the TCM group and blank control group ( $\mathrm{RR}=0.14,95 \% \mathrm{CI}: 0.01$ to $2.59, \mathrm{P}=0.18$ ) (Figure 5).

\section{Gallbladder wall thickness}

Six RCTs, involving 563 cases, compared gallbladder wall thickness between patients who received TCM and patients who received a blank control. Meta-analysis using the 
Table 3 Results of bias risk assessment included in the study

\begin{tabular}{|c|c|c|c|c|c|c|c|}
\hline Study ID & $\begin{array}{l}\text { Sequence } \\
\text { generation }\end{array}$ & $\begin{array}{c}\text { Allocation } \\
\text { concealment }\end{array}$ & $\begin{array}{c}\text { Blinding of } \\
\text { participants and } \\
\text { personnel }\end{array}$ & $\begin{array}{l}\text { Blinding of } \\
\text { outcome } \\
\text { assessors }\end{array}$ & $\begin{array}{c}\text { Incomplete } \\
\text { outcome data }\end{array}$ & $\begin{array}{l}\text { Selective } \\
\text { outcome } \\
\text { reporting }\end{array}$ & $\begin{array}{l}\text { Other } \\
\text { bias }\end{array}$ \\
\hline Wang 2010 (19) & Low risk & Unclear & Unclear & Unclear & High risk & Low risk & Unclear \\
\hline Li 2007 (20) & Low risk & Unclear & Unclear & Unclear & Low risk & Low risk & Unclear \\
\hline Guo 2008 (22) & High risk & Low risk & High risk & Unclear & Low risk & Low risk & Unclear \\
\hline Su 2012 (23) & Low risk & Unclear & Unclear & Unclear & Low risk & Low risk & Unclear \\
\hline Xu 2013 (24) & Low risk & Unclear & Unclear & Unclear & Low risk & Low risk & Unclear \\
\hline
\end{tabular}

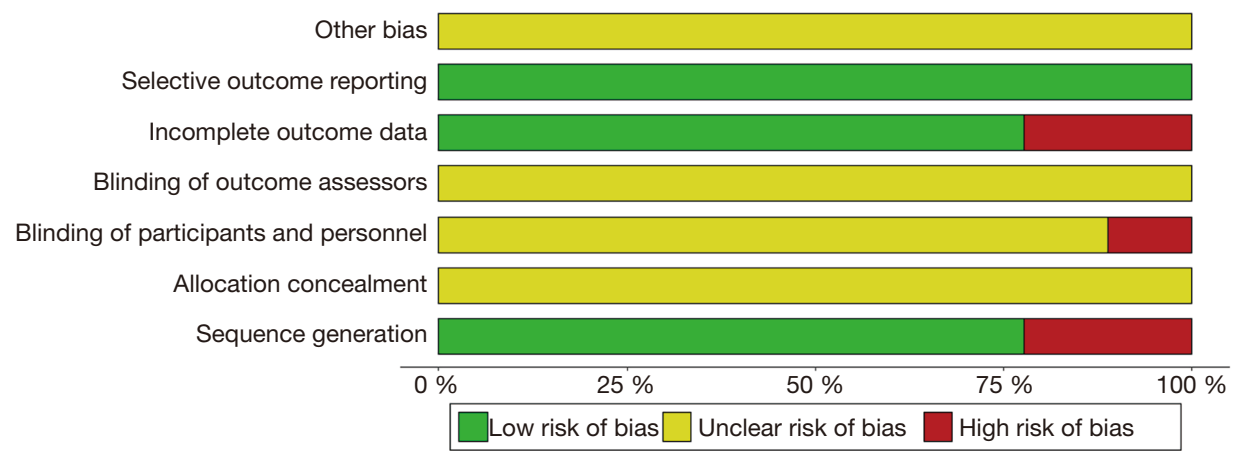

Figure 2 Summary map of bias risk for the included studies.

$\begin{array}{lc}\text { Study } & \text { Experimental group intervention } \\ \text { Zhang 2010 } & \text { Chinese medication } \\ \text { Wang 2010 } & \text { Chinese medication } \\ \text { Li 2007 } & \text { Chinese medication } \\ \text { Liu 2012 } & \text { Chinese medication } \\ \text { Guo 2008 } & \text { Chinese medication } \\ \text { Su 2012 } & \text { Chinese medication } \\ \text { Xu2013 } & \text { Chinese medication } \\ \text { Zhuo 2006 } & \text { Chinese medication } \\ \text { Wang 2016 } & \text { Chinese medication }\end{array}$
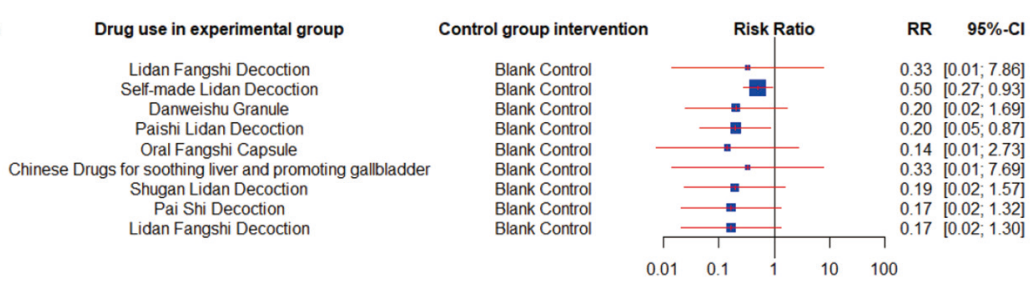

Figure 3 Preventive effect of specific interventions on recurrence. RR, relative risk; CI, confidence interval.

random-effects model showed that there was a significant difference between the TCM group and blank control group (MD $=-0.89,95 \%$ CI: -1.23 to $-0.54, \mathrm{P}<0.001$ ) (Figure 6).

\section{Degree of gallbladder contraction}

Six RCTs, involving 563 cases, compared the degree of gallbladder contraction between patients who received
TCM and patients who received a blank control. Metaanalysis using the fixed-effects model revealed a significant difference between the TCM group and blank control group ( $\mathrm{MD}=14.37,95 \% \mathrm{CI}: 8.00$ to $20.74, \mathrm{P}<0.001$ ) (Figure 7).

\section{Safety}

Two RCTs, involving 91 cases, compared safety between 


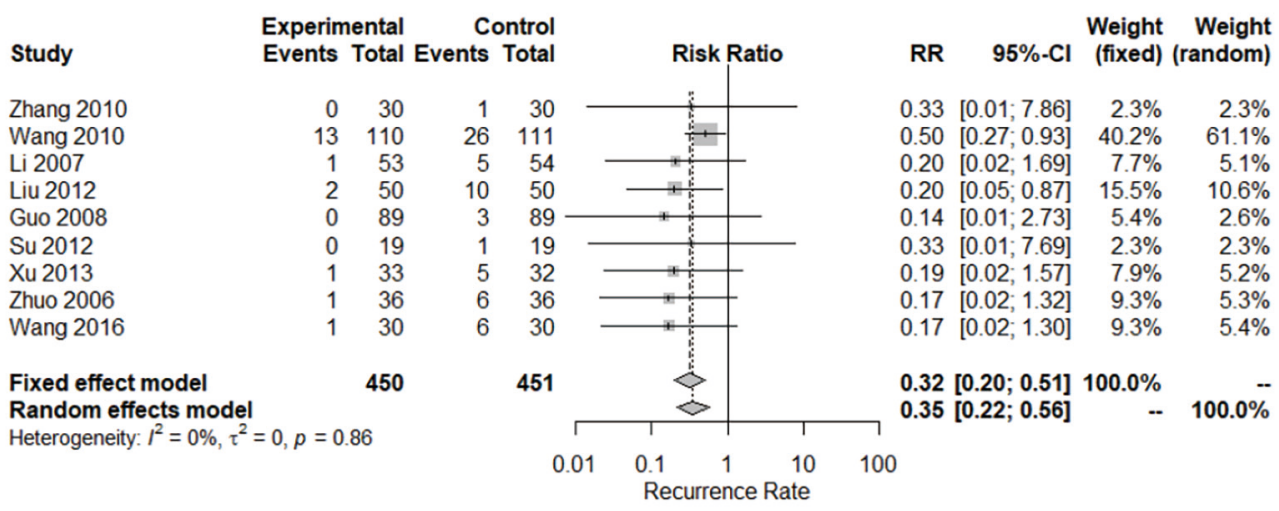

Figure 4 Meta-analysis of recurrence events between the TCM group and blank control group. TCM, traditional Chinese medicine; RR, relative risk; CI, confidence interval.

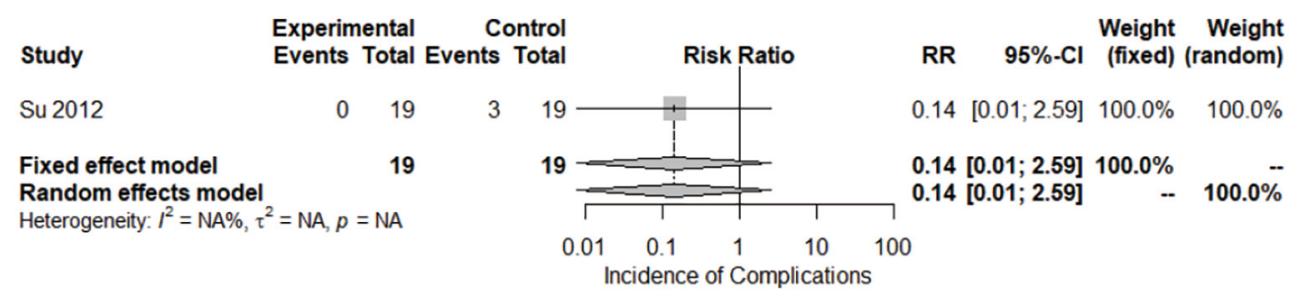

Figure 5 Meta-analysis of the complication morbidity between the TCM group and blank control group. TCM, traditional Chinese medicine; RR, relative risk; CI, confidence interval.

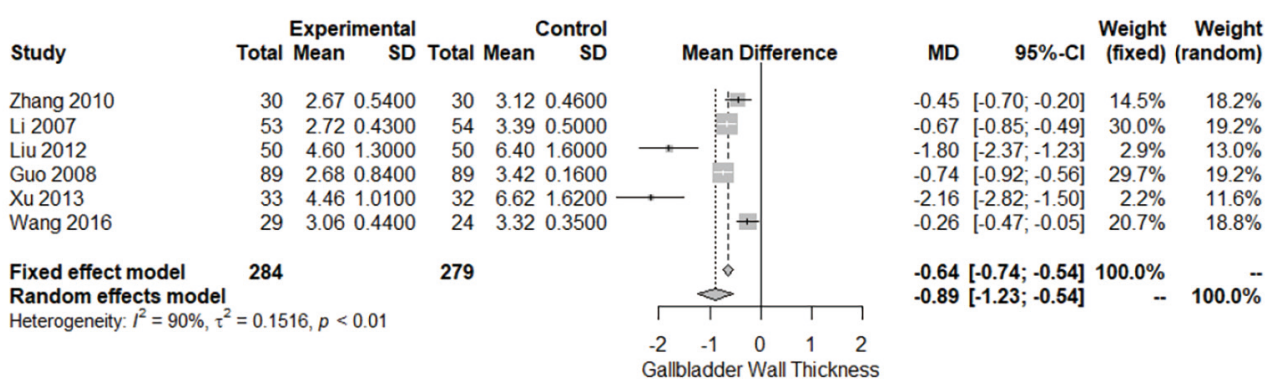

Figure 6 Meta-analysis of gallbladder wall thickness between the TCM group and blank control group. TCM, traditional Chinese medicine; MD, mean difference; CI, confidence interval; SD, standard deviation.

patients who received TCM and patients who received a blank control. Meta-analysis using the random-effects model revealed no significant difference between the TCM group and blank control group (RD $=-0.07,95 \% \mathrm{CI}:-0.26$ to $0.13, \mathrm{P}=0.51$ ) (Figure 8 ).

\section{Sensitivity analysis results}

Sensitivity analysis of the recurrence rate of cholelithiasis after gallbladder-preserving surgery was carried out by comparing TCM with a blank control, which showed no change in the direction of combined results (Figure 9).

\section{Publication bias}

Publication bias test

The recurrence rate of gallstones after gallbladder-preserving 


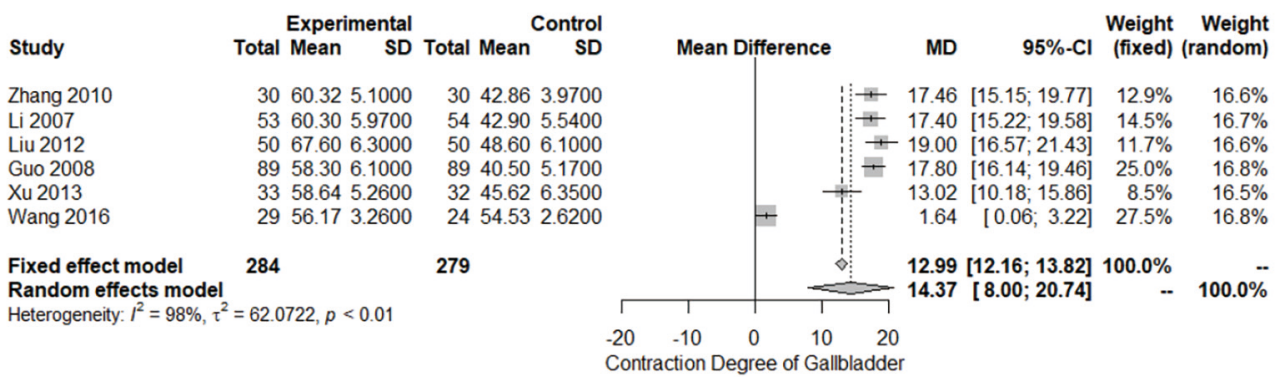

Figure 7 Meta-analysis of gallbladder contraction between the TCM group and blank control group. TCM, traditional Chinese medicine; $\mathrm{MD}$, mean difference; CI, confidence interval; SD, standard deviation.

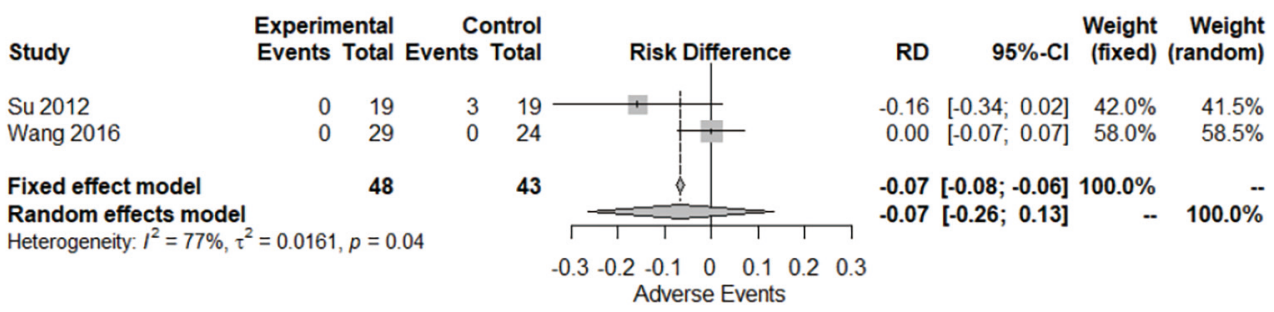

Figure 8 Meta-analysis of safety between the TCM group and blank control group. TCM, traditional Chinese medicine; CI, confidence interval; RD, risk difference.

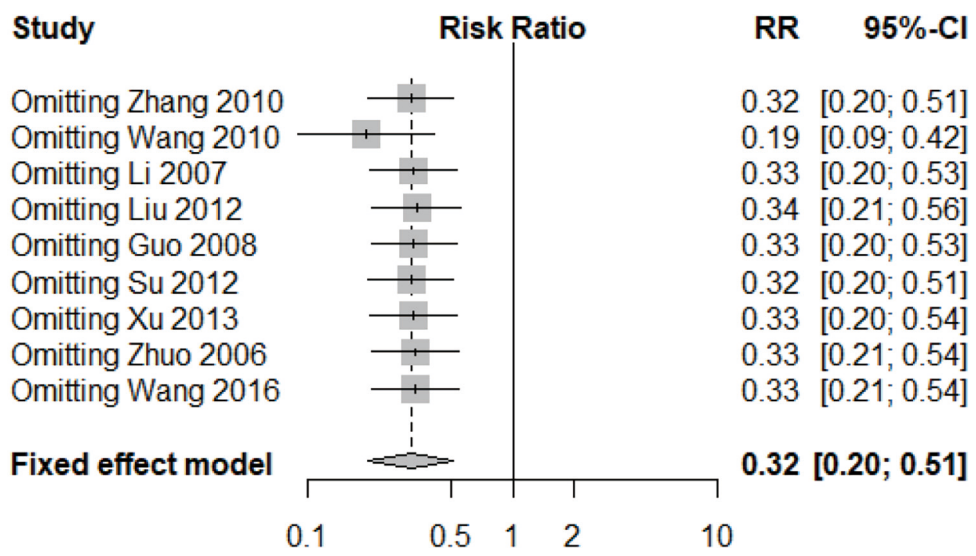

Figure 9 Sensitivity analysis of recurrence events in the TCM group and blank control group. TCM, traditional Chinese medicine; RR, relative risk; CI, confidence interval.

lithotripsy was compared with that of the blank control group by publication bias testing. The funnel diagram shows an asymmetric distribution, suggesting publication bias (Figure 10).

\section{Cut-and-fill method results}

After the addition of 5 more studies, meta-analysis of the recurrence rate using the fixed-effects model revealed a significant difference between the TCM group and the blank control group ( $\mathrm{RR}=0.46,95 \% \mathrm{CI}: 0.30$ to 0.70 , $\mathrm{P}<0.01$ ) (Figure 11). The funnel diagram had a basically symmetrical distribution after pruning, suggesting no significant publication bias (Figure 12). No directional change occurred in the fixed-effects model for recurrence 


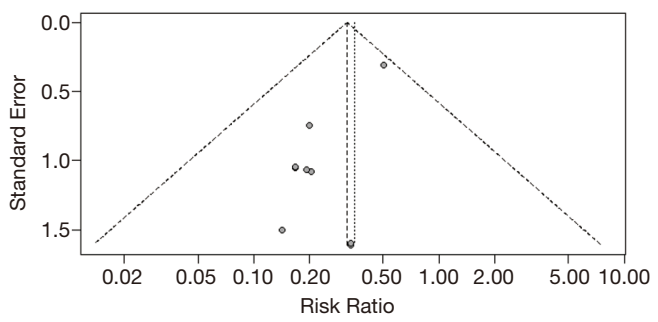

Figure 10 Funnel diagram for the comparison of recurrence events between the traditional Chinese medicine group and blank control group.

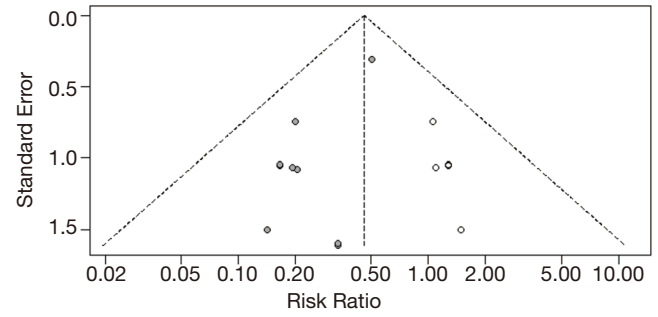

Figure 12 Funnel diagram showing recurrence events in the TCM group and blank control group after clipping and tonifying. TCM, traditional Chinese medicine.

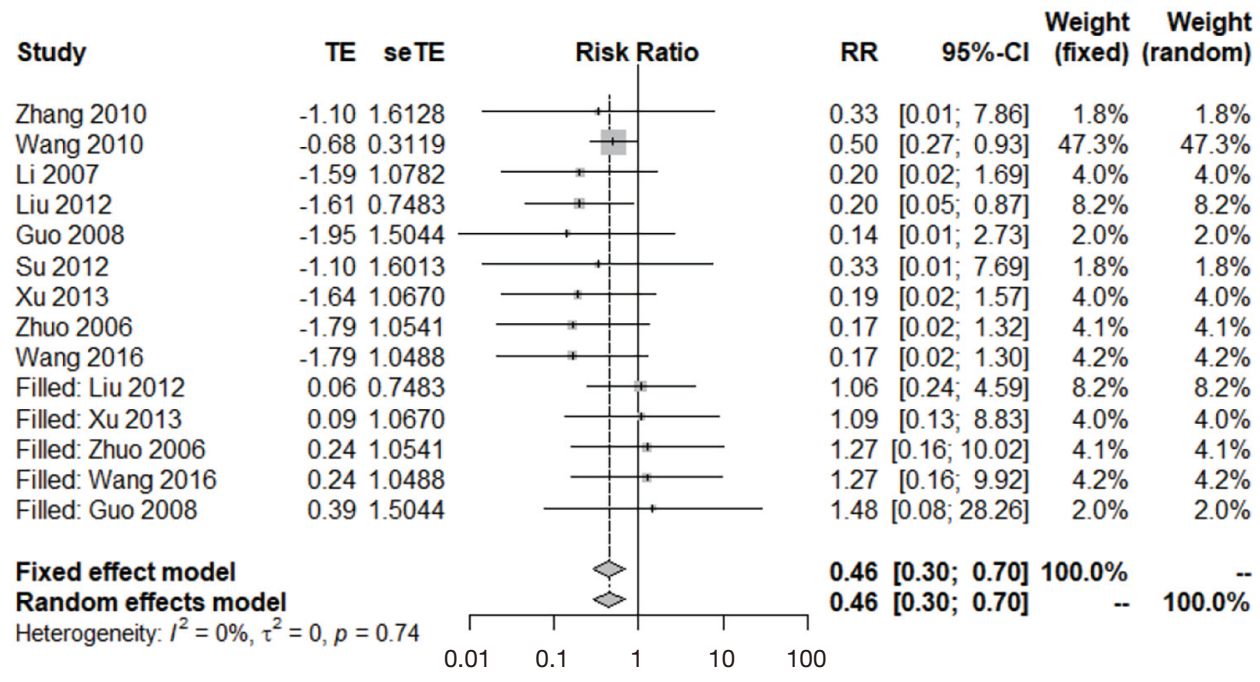

Figure 11 Meta-analysis of recurrence events between the TCM group and blank control group after pruning. TCM, traditional Chinese medicine; RR, relative risk; CI, confidence interval.

rate before and after pruning, suggesting the stability of this study's results.

\section{Evidence quality evaluation based on the GRADE system}

The recurrence rate was the critical outcome index, and the incidence of complications, gallbladder wall thickness, gallbladder wall contraction degree, and rate of adverse reactions were the important outcome indexes. Table 4 displays a summary of evidence based on the GRADE system.

\section{Analysis of intervention on specific use of TCMs}

\section{Specific use of TCM}

As shown in Table 5, TCMs used at least 5 times across all studies $(>50 \%)$, were included as specific TCMs in pathway analysis.

\section{Disease-related genes included in the intervention of active ingredients of TCM}

We retrieved hits of the active ingredients of TCMs and regulatory gene targets. Disease-related genes were also retrieved and incorporated. The disease-related genes that were intersected and included in the intersection of active ingredients of TCM were collected and are summarized in the Wayne diagram in Figure 13.

\section{Effective components of TCM and disease-related gene targets}

The sources of active TCM ingredients and intervention targets are shown in Table 6 , and the active ingredient-target 


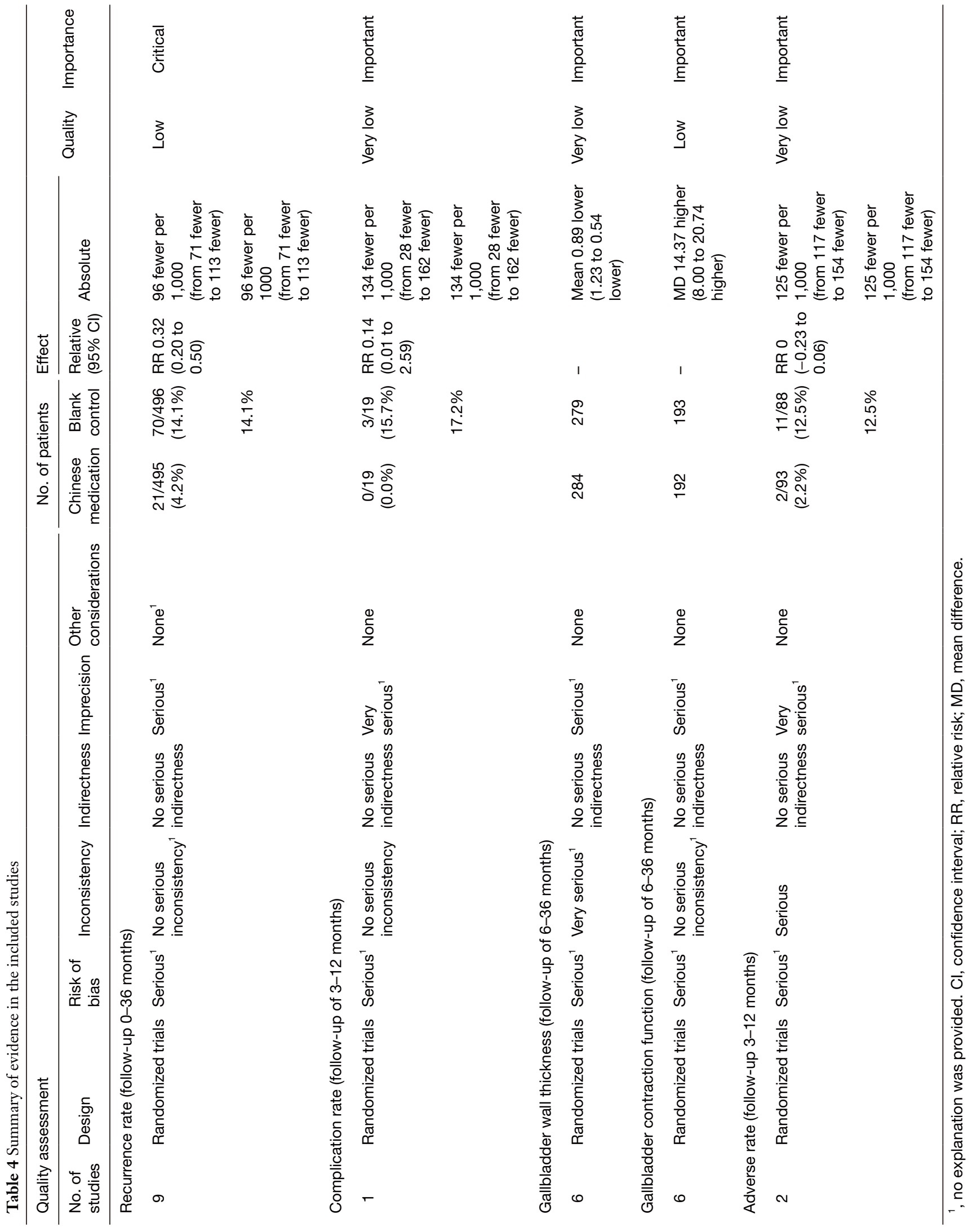


Table 5 Chinese medicines reused in different studies included in meta-analysis (unit: g)

\begin{tabular}{|c|c|c|c|c|c|c|c|c|}
\hline Study ID & $\begin{array}{l}\text { Lysimachia } \\
\text { christinae }\end{array}$ & $\begin{array}{c}\text { Radix } \\
\text { Bupleuri }\end{array}$ & Curcuma & Licorice & Bitter orange & $\begin{array}{l}\text { Paeonia } \\
\text { lactiflora }\end{array}$ & $\begin{array}{c}\text { Chicken } \\
\text { gallbladder }\end{array}$ & $\begin{array}{l}\text { Chinese } \\
\text { rhubarb }\end{array}$ \\
\hline Zhang 2010 (18) & 30 & 10 & 10 & 10 & & 20 & 10 & 10 \\
\hline Li 2007 (20) & 15 & 15 & 15 & 5 & & 20 & & \\
\hline Su 2012 (23) & 15 & 10 & 10 & & & 10 & & \\
\hline Xu 2013 (24) & 30 & 10 & 10 & 10 & & & 10 & 10 \\
\hline Zhuo 2006 (25) & 30 & 10 & 10 & 5 & 10 & 15 & 10 & 10 \\
\hline
\end{tabular}

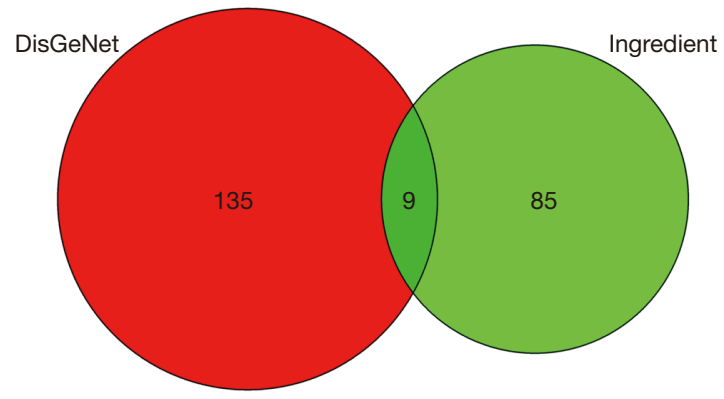

Figure 13 A summary of genes associated with gallstone disease intersected with active ingredients of TCM. TCM, traditional Chinese medicine.

network is shown in Figure 14. Among them, catechin, stigmatin, kaempferol, naringin, and quercetin are the effective components that regulate the genes related to gallstone disease.

\section{Annotation analysis of specific TCM pathways}

The results of enrichment analysis with GO functional annotations the target genes of TCMs (Figure 15) suggested that for gallstones, the effects of TCMs are related to nuclear receptor activity $(\mathrm{P}<0.001)$, transcription factor activity $(\mathrm{P}<0.001)$, sequence-specific DNA binding $(\mathrm{P}<0.001)$, steroid hormone receptor activity $(\mathrm{P}<0.001)$, core promoter sequence-specific DNA binding $(\mathrm{P}<0.001)$, steroid binding $(\mathrm{P}<0.001)$, core promoter binding $(\mathrm{P}<0.001)$, RNA polymerase II proximal promoter sequence-specific DNA binding $(\mathrm{P}<0.001)$, proximal
Table 6 Sources of active components of drugs and targets for intervention

\begin{tabular}{lllc}
\hline Symbol & Type & Source & Count \\
\hline (-)-catechin & Herb & Dahuang & 1 \\
Formononetin & Herb & Gancao & 3 \\
Kaempferol & Herb & Chaihu & 2 \\
& Herb & Gancao & \\
Naringenin & Herb & Gancao & 4 \\
Quercetin & Herb & Gancao & 5 \\
& Herb & Chaihu & \\
\hline
\end{tabular}

promoter sequence specific DNA binding $(\mathrm{P}<0.001)$, nuclear hormone receptor binding $(\mathrm{P}<0.001)$, hormone receptor binding $(\mathrm{P}<0.001)$, and other biological processes. The KEGG pathway enrichment analysis results are shown in Figure 16. They suggested that for gallbladder stones, the effects of TCMs are related to proteoglycan $(\mathrm{P}=0.02)$, ovarian steroid production $(\mathrm{P}=0.02)$, cholesterol metabolism $(\mathrm{P}=0.02)$, renal cell carcinoma $(\mathrm{P}=0.03)$, the prolactin signaling pathway $(\mathrm{P}=0.03)$, endocrine resistance $(\mathrm{P}=0.04)$, and the HIF-1 signaling pathway $(\mathrm{P}=0.04)$. As shown in Figure 17, it was found that the regulatory mechanism of TCMs on gallstones may be related to the cholesterol metabolic pathway. We found that the effects of TCMs are also related to the regulation of apolipoprotein B and low-density lipoprotein (LDL) receptor genes. 


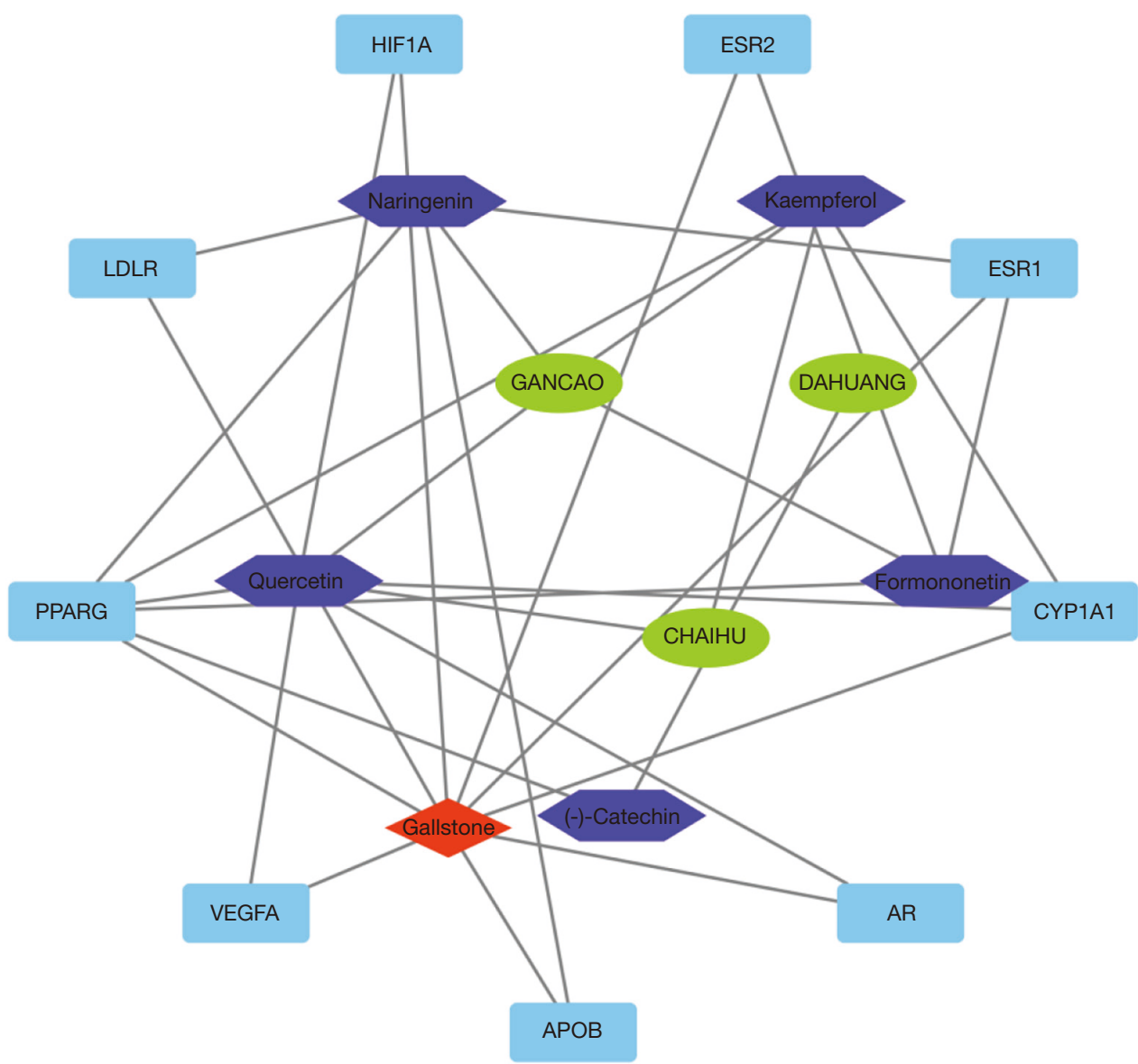

Figure 14 Drug active component-target network.

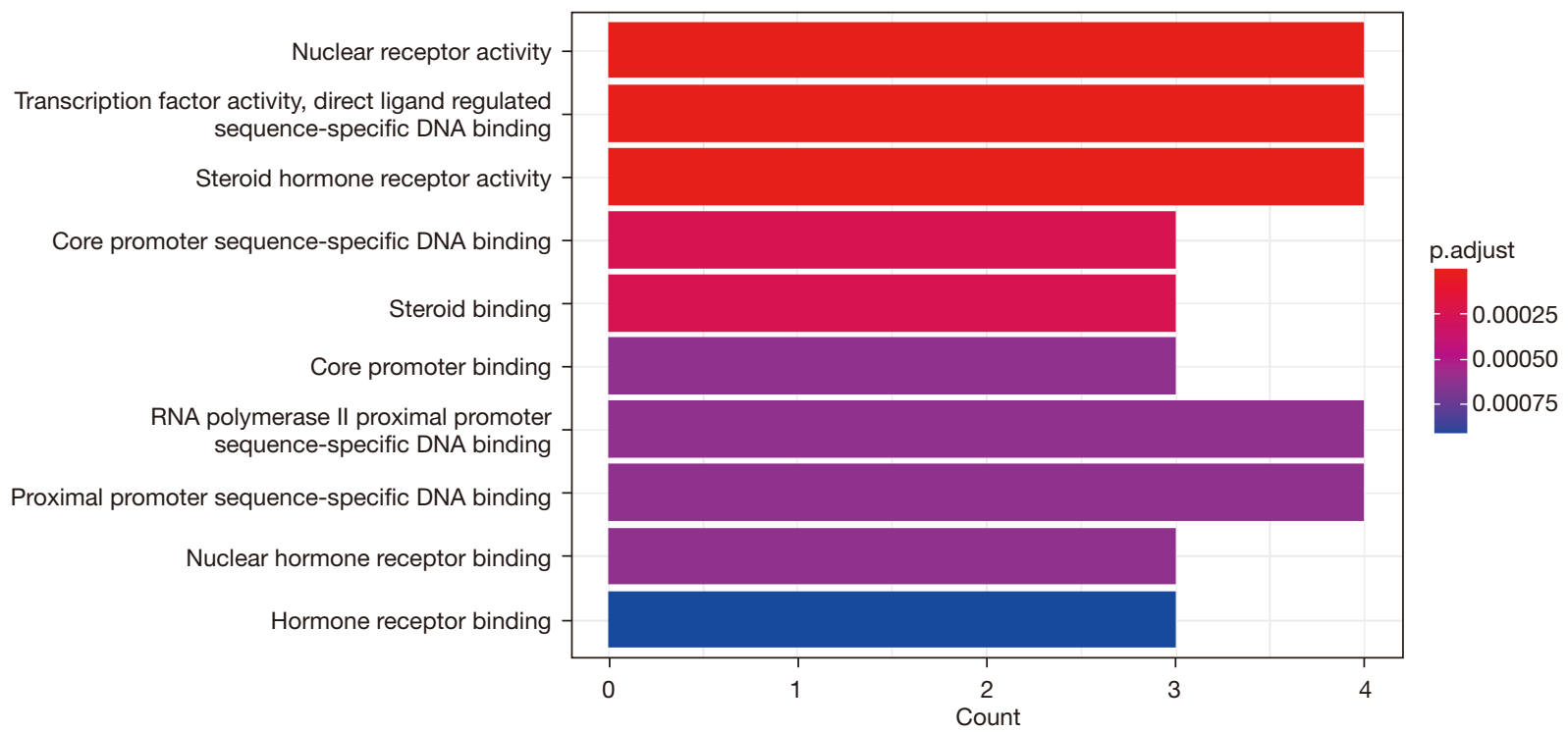

Figure 15 Enrichment and analysis results of GO functional notes of TCMs for preventing gallstone recurrence. GO, Gene Ontology; TCM, traditional Chinese medicine. 


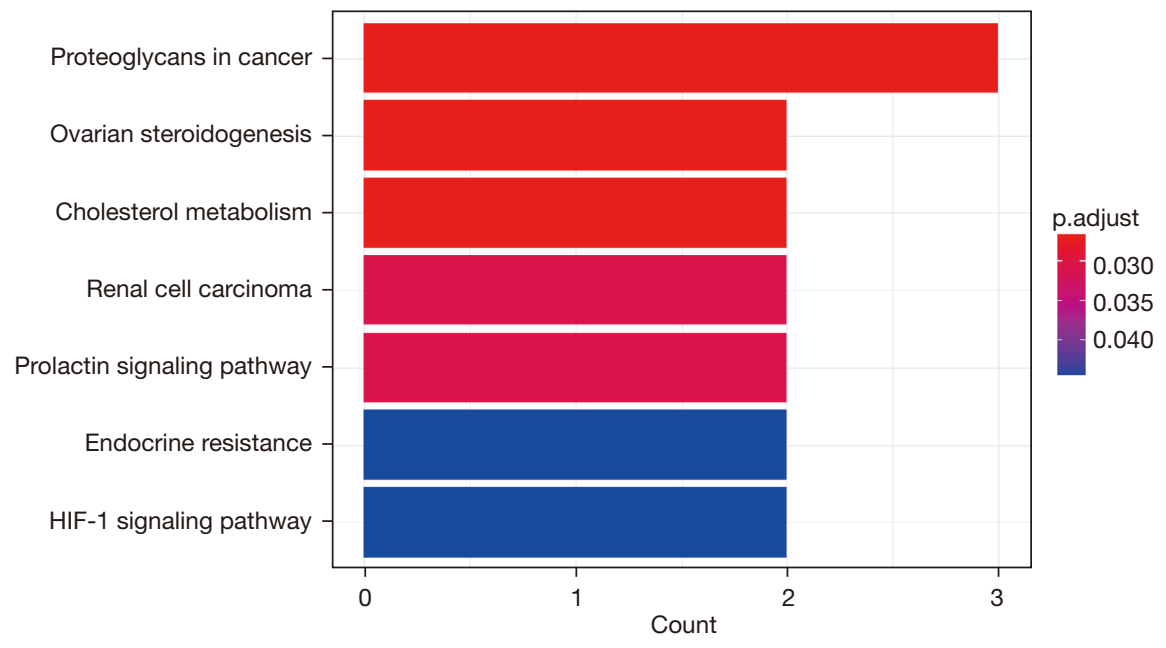

Figure 16 Enrichment analysis of TCM-related KEGG pathways in the prevention of gallstone recurrence. TCM, traditional Chinese medicine; KEGG, Kyoto Encyclopedia of Genes and Genomes.

\section{Discussion}

This study included 9 RCTs in a meta-analysis for systematic review (SR). In the results of the meta-analysis for gallbladder wall thickness, degree of gallbladder contraction and safety, the I 2 were greater than $75 \%$. That may be caused by the significantly different results of the include studies. But we did not find significant differences in trial setting between the included studies as well as a significant reason for heterogeneity. Hence, to prevent information loss and present the real effect of TCM healthcare, we determined to keep the results with high heterogeneity instead of excluding possible studies that caused heterogeneity.

The main findings were as follows: (I) the recurrence rate and the incidence of complications in the TCM group were reduced compared to those in the blank control group. This finding suggested that TCM might have a preventive effect on disease recurrence and related complications following gallbladder-preserving surgery, and it could reduce the recurrence rate by about $10 \%$. (II) The thickness of the gallbladder wall and the degree of gallbladder contraction in the TCM group were less than those in the blank control group, suggesting that TCM may improve the physiological function of gallbladder after gallbladder-preserving lithotripsy.

The basic factors of gallbladder stones are supersaturation of cholesterol (27) caused by abnormal cholesterol transport in the liver, and influenced by local environmental factors of the gallbladder (27): (I) nuclear factors contributing to gallbladder secretion include excess secretion/accumulation of mucin (28) and fibronectin (29); (II) the dynamics of gallbladder cholestasis include: decreased sensitivity of cholecystokinin and decreased basic tension of the gallbladder. Increases in the fasting volume and residual index of the gallbladder result in abnormal gallbladder emptying and cholestasis, which eventually lead to gallstone formation (30). Cholesterol-supersaturated bile and a gallbladder environment which is beneficial to the development of bile crystallization can also easily lead to gallstone recurrence (27). Current evidence-based medical findings suggest that the main risk factors for recurrence of cholelithiasis after gallbladder-preserving surgery are a family history of gallstones, multiple gallstones, gallbladder wall thickening, a dietary preference for greasy food, gallbladder dysfunction, and failure to take ursodeoxycholic acid after the surgery (31).

The TCMs for maintaining the integrity of the gallbladder which featured in this study mainly involve herbs such as Lysimachia chrysanthemum, Bupleurum, Tulip, and Fructus Aurantii, and prevent stone recurrence by regulating bile cholesterol supersaturation and the gallbladder environment. Fructus Aurantii can reduce the serum cholesterol level, thereby regulating the supersaturation of bile with cholesterol (32); Bupleurum Saikosaponin-d in Bupleurum can inhibit fibronectin secretion, thereby regulating the gallbladder local environment (33); Tulip can constrict gallbladder smooth muscle and inhibit the contractile activity of the sphincter of Oddi (34); and Lycopodium mongolicum improves 


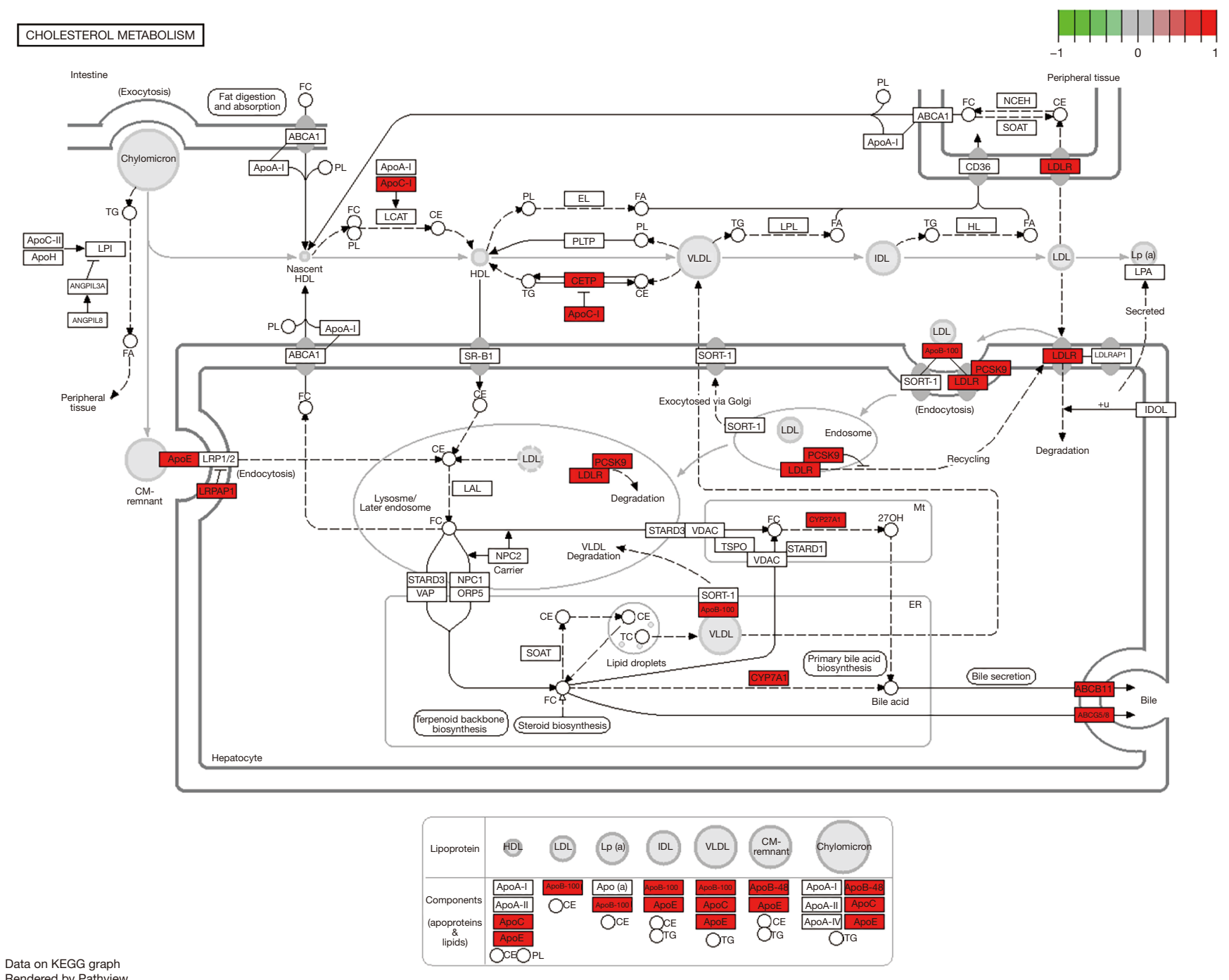

Figure 17 Cholesterol KEGG pathway map. KEGG, Kyoto Encyclopedia of Genes and Genomes.

the serum cholecystokinin receptor level (35). These pharmacological effects may be related to the mechanism of preventing gallstone recurrence after gallbladder-preserving surgery with TCM.

Combined with the characteristics of gallstones, the preventative and regulatory mechanism of TCMs on gallstones may be related to the cholesterol metabolic pathway. Naringin from Glycyrrhiza uralensis can regulate apolipoprotein B and LDL receptor-related genes of gallstone disease to intervene in disease progression. The possible mechanism is that apolipoprotein $\mathrm{B}$, which is expressed by the apolipoprotein B gene and contains very low density lipoprotein (VLDL), medium-density lipoprotein (IDL), and LDL (36), transports cholesterol in the blood and participates in the regulation of cholesterol supersaturation of bile. LDL receptors can transform cholesterol into bile acids, steroids, and vitamin D3 by specifically recognizing apolipoprotein binding to LDL $(37,38)$, possibly via naringin. The mechanism of regulating the supersaturation of bile with cholesterol to prevent gallstone recurrence requires elucidation through further basic experimental verification.

The treatment of gallstones with TCM involves the principle of "soothing the liver and regulating qi, promoting the gallbladder, and removing stones" (39). It is upheld in TCM that gallstones belong to the syndrome of liver failure 
and diarrhea, and damp-heat blockade. The corresponding herbal formulae used to treat gallstones include Dachaihu Tang, Chaihu Shugan San, and Haoqin Qingdan Tang. Another perspective is presented in the Treatise on Febrile Diseases, which ascribes gallstones to the category of Taiyin disease. The prescription Sinisan is also effective in the treatment of gallstones. Most of the TCMs selected in this paper are compositions of the above prescriptions.

In terms of the stone recurrence rate, a similar study (11) previously showed that there was a significant difference in non-recurrence events between the TCM group and the control group ( $\mathrm{RR}=1.09$, $\mathrm{CI}: 1.04$ to $1.14, \mathrm{P}<0.001]$. According to the original data, the difference in recurrence events between the TCM group and the blank control group was calculated as follows: $(\mathrm{RR}=0.32,95 \% \mathrm{CI}$ : 0.18 to $0.57, \mathrm{P}<0.01)$. As this analysis only involved studies which included a pure TCM experimental group and blank control group, the expansion effect of other therapeutic factors on the efficacy of TCM was reduced.

The evidence quality for the critical outcome indicator of recurrence rate, evaluated using the GRADE system, was "low". The quality of evidence for important outcome indicators was as follows: incidence of complications: "very low"; gallbladder wall thickness: "very low"; gallbladder wall contraction degree: "low", and adverse reaction rate: "very low". The main reasons for the deterioration of the evidence quality of outcome indicators were the risk bias of the included studies and the inaccuracy of data merging results, which resulted from the lack of hidden grouping, the lack of blind experimental design, and the CI of data merging results being too wide owing to the small number of samples included in the experiment $(40,41)$.

The limitations of this study were as follows. First, the quality of the included research was not high, and most articles did not describe specific random methods, or mention allocation concealment or blinding methods, which would have seriously affected the conclusions of the study. Second, the studies included were RCTs, but the strict inclusion exclusion criteria may have limited the extrapolation of study results. Third, all included studies were performed in China, and no English literatures that met the inclusion criteria were retrieved, which may limit the applicability of the meta-analysis results. Finally, some results of meta-analysis were with high heterogeneity, which may lead to an unrobust conclusion.

The results of this meta-analysis suggest that more clinical trials based on strict randomization, a large sample size, and double blindness should be carried out in the future to advance the prevention of recurrence of cholelithiasis after gallbladder-preserving surgery using TCM. At the same time, the clinical efficacy of Wendan Decoction and other commonly used prescriptions in the treatment of phlegm syndrome should be verified (42). The existing evidence shows that TSM can reduce the recurrence rate of cholelithiasis and improve the physiological function of the gallbladder after cholelithotomy; therefore, it can be considered for clinical use. Due to the limitations of the quantity and quality of the included studies, more highquality studies are needed to support the above conclusions.

\section{Acknowledgments}

Funding: The study was supported by the National Natural Science Foundation of China (81774146) and the Beijing NOVA Programme (xxjh2015A093; Z1511000003150125).

\section{Footnote}

Provenance and Peer Review: This article was commissioned by the editorial office, Annals of Palliative Medicine for the series "Narrative \& Evidence-based Medicine for Traditional Medicine: from basic research to clinical practice and trial". The article has undergone external peer review.

Reporting Checklist: The authors have completed the PRISMA reporting checklist. Available at https://dx.doi. org/10.21037/apm-20-1135

Conflicts of Interest: All authors have completed the ICMJE uniform disclosure form (available at https://dx.doi. org/10.21037/apm-20-1135). The series "Narrative \& Evidence-based Medicine for Traditional Medicine: from basic research to clinical practice and trial" was commissioned by the editorial office without any funding or sponsorship. BL served as the unpaid Guest Editor of the series. The authors have no other conflicts of interest to declare.

Etbical Statement: The authors are accountable for all aspects of the work in ensuring that questions related to the accuracy or integrity of any part of the work are appropriately investigated and resolved.

Open Access Statement: This is an Open Access article distributed in accordance with the Creative Commons 
Attribution-NonCommercial-NoDerivs 4.0 International License (CC BY-NC-ND 4.0), which permits the noncommercial replication and distribution of the article with the strict proviso that no changes or edits are made and the original work is properly cited (including links to both the formal publication through the relevant DOI and the license). See: https://creativecommons.org/licenses/by-nc-nd/4.0/.

\section{References}

1. Wang Y, Xu HW. Risk Factors of Cholelithiasis in China: a Meta-analysis. Chin J Hepatobiliary Surg 2016;22:386-90.

2. Ouyang YF, Wang HJ, Su C, et al. Changes of BMI Distribution in Chinese Adults from 1989 to 2011. Acta Nutr Sin 2014;36:529-34.

3. Zhao L, Fang Y, He Y, et al. Trends of food consumption among Chinese population in 1992-2012. Wei Sheng Yan Jiu 2016;45:522-6.

4. Lu CN, Min YX, Yu GW. Progress in Epidemiological Study of Cholelithiasis among Ethnic Minorities in China. Chi Prim Health Care 2009;23:80-1.

5. Zeng Q, He Y, Qiang DC, et al. Prevalence and epidemiological pattern of gallstones in urban residents in China. Eur J Gastroenterol Hepatol 2012;24:1459-60.

6. Tazuma S, Unno M, Igarashi Y, et al. Evidence-based clinical practice guidelines for cholelithiasis 2016. J Gastroenterol 2017;52:276-300.

7. Zhi XT. My Opinion on Gallbladder Preservation and Stone Removal. J Laparosc Surg 2009;14:12-4.

8. Zhang BS. Discussion on the New Thinking of Minimally Invasive Laparoscopic Cholecystolithotomy. J Laparosc Surg 2009;21:337-40.

9. Zhang BS. Endoscopic Minimally Invasive Cholecystolithotripsy for Gallstones. China J Endosc 2002;8:1-4.

10. Nursing Experience of 65 Cases after Laparoscopic Choledochoscopic Cholelithotripsy. World Latest Med Inf 2015;15:174-5.

11. Li ZJ, Wang XK, Guan XW. Meta-analysis of the Efficacy of Traditional Chinese Medicine in Preventing Recurrence of Gallstones after Minimally Invasive Cholecystolithotomy. Mod Diagn Treat 2015;26:3640-1.

12. Editor Board Committee of Chinese Journal of Disgetion. Chinese Consensus on the Medical Diagnosis and Treatment of Chronic Cholecystitis and Gallstone (2014, Shanghai). J Clin Hepatol 2015;35:292-6.

13. Higgins JPT, Altman DG, Gøtzsche PC, et al. The Cochrane Collaboration's tool for assessing risk of bias in randomised trials. BMJ 2011;343:d5928.

14. Higgins JPT, Altman DG. Chapter 8:assessing risk of bias in included studies [online]. Available online: https:// training.cochrane.org/handbook/current/chapter-08 [Accessed 22 May 2021]

15. Guyatt GH, Oxman AD, Vist GE, et al. GRADE: an Emerging Consensus on Rating Quality of Evidence and Strength of Recommendations. BMJ 2008;336:924.

16. Howard B, Mark H, Schünemann HJ, et al. GRADE guidelines:3. Rating the quality of evidence. J Clin Epidemiol 2011;64:401-6.

17. Balshem H, Helfanda M, Schunemann HJ, et al. GRADE Guidelines:3. Rating the Quality of Evidence. Chin J Evid Based Med 2011;11:451-5.

18. Zhang P, Li Q, Liu L, et al. Preventative Effect of Lidanfangshi Powder On Relapse of Lithiasis after Microinvasive Gallbladder-protected Llthotomy. Chin Gen Pract 2010;13:991-3.

19. Wang F, Zhang J, Gao Y, et al. Observation on the Effect of Lidan Decoction in Preventing the Recurrence of Cholelithiasis after Lithotomy. J Gansu Univ Chin Med 2010;27:18-20.

20. Li J, HuangM, Lu Y, et al. editors. Clinical Observation Study of Danweishu Granule in Preventing Recurrence of Gallstone after Lithotomy. Found Conf First Annu Meet Branch Integr Tradit Chin West Med Chin Med Assoc 2007:138-44.

21. Liu YF, Zhang C, Li XY. Clinical Observation Study of Lidan Paishi Decoction in Preventing Stone Recurrence after Gallbladder-Preserving Lithotripsy. China Health Ind 2012;9:165-7.

22. Guo SJ, Li J, Zhang JC, et al. Clinical Study on Prevention of Gallstone Recurrence by Fangshi Capsule. Chin J Integr Tradit West Med Dig 2008;16:254-5.

23. Su JC, Su JC, W L. The effect of choledochoscope calculus removed plus postoperative herbal interna. China Mod Dr 2012;50:64-65,69.

24. Xu YZ, Wang YJ, Wang AH. Observation on Effect of Shugan Lidan Decoction on Complications after Gallbladder-Preserving Lithotripsy. Shaanxi J Tradit Chin Med 2013;34:992-4.

25. Zhuo XY, Luo LY, Liu B. Clinical Observation Study and Nursing of Using Paishi Decoction to Prevent Recurrence of Cholelithiasis after Minimally Invasive Cholelithotomy. J Clin Res 2006;23:1329-30.

26. Wang ZY. Clinical Study of Lidan Fangshi Decoction on Prevention of Gallstone Recurrence after Minimally Mvasuve Lithotomy, Master Thesis, China: Guiyang 
College of Traditional Chinese Medicine 2016.

27. Han TQ, Zhang SD. Recurrence and Prevention of Gallstones after Gallstone-Preserving Operation. Chin J Hepatobiliary Surg 2009; 15:4-5.

28. Levy PF, Smith BF, LaMont JT. Human gallbladder mucin accelerates nucleation of cholesterol in artificial bile. Gastroenterology 1984;87:270-5.

29. Chijiiwa K, Koga A, Yamasaki T, et al. Fibronectin: a possible factor promoting cholesterol monohydrate crystallization in bile. Biochim Biophys Acta 1991;1086:44-8.

30. Zhu MY, Wang ZW, Chen JP, et al. The Expression of CCK mRNA of Gallbladder Wall in 100 Paitents with Gallstones. J HPB Surg 2004;16:255-7.

31. Li W, Huang P, Lei P, et al. Risk factors for the recurrence of stones after endoscopic minimally invasive cholecystolithotomy in China: a meta-analysis. Surg Endosc 2019;33:1802-10.

32. Wu LH, Ji ZQ, Ji WY, et al. The Influence of Fructus Aurantii Immaturus on Blood Lipids and Vasular Endothelial Function in Paients with Hyperlipidemia. Chin J Integrative Med Cardio Cerebrovasc Dis 2012;10:283-5.

33. Lu CN. Anti-Inflammatory Activities and Effects on the Signal Transduction Pathway of NF-kappa B of Saikosaponins A and D, Master Thesis, China: South China Agricultural University; 2012.

34. Wang LD, Li HF. Effects of Single Tulip on Smooth Muscle Activity of Isolated Rabbit Oddie's Sphincter, Gallbladder and Duodenum. J Gansu Coll Tradit Chin Med 2002;19:14-5.

Cite this article as: Li B, Lin Y, Nie B, Zhao G, Zhang X, Zhu M, Hu J, Huang P, Feng S, Qiu R, Tian G, Zhang S, Shang H. Systematic review and pathway enrichment analysis of Chinese medicine in preventing recurrence and improving prognosis of cholelithiasis after gallbladder-preserving lithotripsy. Ann Palliat Med 2021;10(12):12985-13001. doi: 10.21037/apm-20-1135
35. Wu HJ, Li CL, Ma CL, et al. Effects of Herba Lysimachiae on Serum Cholecystokinin Receptor and Vasoactive Intestinal Peptide Levels in Patients with Cholecystolithiasis. Mod J Integr Tradit Chin West Med 2017;26:2793-5.

36. Feingold KR, Grunfeld C. Introduction to Lipids and Lipoproteins[online]. Available online: https://www. endotext.org/chapter/introduction-to-lipids-andlipoproteins/ [Accessed 25 May 2021]

37. Brown MS, Goldstein JL. How LDL receptors influence cholesterol and atherosclerosis. Sci Am 1984;251:58-66.

38. Long XJ, Yin RX. Research Progress on the Relationship between Low Density Lipoprotein Receptor Gene and Serum Cholesterol. Med Inf 2010;5:1326-8.

39. Professional Committee on Digestive System Diseases of Chinese Association of Integraive Medicine. Consensus on Diagnosis and Treatment of Cholelithiasis with Integrated Traditional Chinese and Western Medicine. Chin J Integr Tradit West Med Dig 2011;31:1041-3.

40. Guyatt GH, Oxman AD, Vist GE, et al. GRADE Guidelines: 4. Rating the Quality of Evidence-study Limitations (Risk of Bias). Chin J Evid Based Med 2011;11:456-63.

41. Guyatt GH, Oxman AD, Kunz R, et al. GRADE guidelines:5. Rating the quality of evidence-publication bias. Chin J Evid Based Med 2011;11:1435-43.

42. Fang YQ, Cao JH, Fang CL. Discussing the Essence of Phlegm from the Syndrome of Wendan Decoction. China J Basic Med Tradit Chin Med 1998;4:43-5.

(English Language Editors: J. Jones and J. Reynolds) 